\title{
Supplementation of non-fermented
} and fermented goji berry (Lycium barbarum) improves hepatic function and corresponding lipid metabolism via their anti-inflammatory and antioxidant properties in high fat-fed rats

Sunhye Lee®l, Soyeon Jeong, Yeongju Park, Hyunji Seo, Cheongbin You, Unsik Hwang, Hoon Park and Hee-jae Suh*

\begin{abstract}
Development of obesity is associated with excessive fat accumulation and oxidative stress along with chronic inflammation. Goji berries (Lycium barbarum) are high in polyphenolic compounds and have anti-inflammatory, anti-oxidant, and hypolipidemic properties that may alleviate the pathogenesis of obesity and related metabolic complications. Thus, the aim of this study was to investigate potential metabolic benefits of GB supplementation against high fat (HF) diet-induced obesity and its comorbidities in HF diet-fed rats (male Sprague-Dawley, $n=8 /$ group, 6 weeks old). We also sought to examine the potential metabolic benefits of fermented GB (FGB) with L. plantarum CB3 and possible distinctions in the degree and/or mechanism of action compared to GB. GB and FGB supplementation suppressed the gene expression of inflammation indices at the local (adipose tissues) and systemic (liver) levels. In addition, GB and FGB supplementation upregulated the gene expression of antioxidant enzymes compared to the HF and/or even low fat (LF) group with more remarkable antioxidant effects by GB supplementation. Also, GB and FGB supplementation protected from HF-induced damages of the liver and dyslipidemia. In conclusion, we demonstrated that GB and FGB supplementation protected from HF-induced metabolic complications primarily by improving hepatic function and corresponding lipid metabolism via their anti-inflammatory and antioxidant properties. To our knowledge, this is the first in vivo study confirming metabolic benefits of $G B$ in a fermented form. Thus, these findings support the potential application of both GB and FGB to ameliorate obesity-associated metabolic abnormalities.
\end{abstract}

Keywords: Dyslipidemia, Fermentation, Goji berry (Lycium barbarum), Hepatic function, Inflammation, Obesity, Oxidative stress

*Correspondence: suhhj@sunmoon.ac.kr

Department of Food Science, Research Center for Food and Bio

Convergence, Sun Moon University, Asan, Chungchengnam-do 31460,

Republic of Korea

\section{Introduction}

Obesity is primarily characterized by abnormal or excessive fat accumulation and a low-level systemic inflammation [1]. An increase in visceral adiposity serves as a strong predictor of local and systemic inflammation with its association with macrophage infiltration and subsequent secretion of pro-inflammatory cytokines [2]. The pivotal role of pro-inflammatory cytokines such as interleukin-1beta (IL-1 $1 \beta$ ) and 
tumor necrosis factor alpha (TNF $\alpha)$ has been identified in the development of obesity and related metabolic dysregulation [3]. Further, the inflammatory cascade can facilitate the formation of toxic reactive oxygen species and the subsequent generation of oxidative stress, which inhibits the expression of antioxidant enzymes and consequently impairs the antioxidant defense system [3]. Excessive oxidative stress also can impair liver functions, in the regulation of hepatic lipid metabolism in particular [4]. Previous studies have reported that HF-induced hepatic dysfunction demonstrated by the elevated level of alkaline phosphatase (ALP) and aspartate transaminase (AST) enzyme activities in circulation was associated with the upregulation of adipogenesis and lipogenesis transcription factors, resulting in hepatic fat accumulation and dyslipidemia $[5,6]$.

Extensive attention has been paid to the role of berries in the prevention of obesity and related metabolic disturbances. Goji berries (Lycium barbarum) are high in polyphenolic compounds and have antiinflammatory, anti-oxidant, and hypolipidemic properties that may affect disease pathogenesis [7]. Dietary supplementation with goji berry (GB) reduced biomarkers of oxidative stress and lipid peroxidation as well as inflammatory gene expression in both experimental and clinical studies [8-10]. In addition, Yang et al. reported that GB supplementation ameliorated HF-induced insulin resistance via activation of phosphatidylinositol-3 kinase/protein kinase B/nuclear factor-E2-related factor 2 (PI3K/AKT/Nrf2) pathway [11].

Despite a growing evidence on metabolic benefits of GB, there is limited knowledge on the potential benefits of GB in obesity and related metabolic disorders. Thus, the aim of this study was to investigate metabolic benefits of GB supplementation against HF diet-induced obesity and its comorbidities in rats. We hypothesized that GB supplementation would improve HF-induced obese phenotypes and dysregulation of lipid metabolism in association with anti-inflammatory and anti-oxidant mechanisms. Further, considering that fermentation of bioactive prebiotics with probiotics such as Lactobacillus or Bifidobacterium has been confirmed to enhance the pharmacological efficacy and metabolic functions of the original nonfermented prebiotics in previous studies [12-14], we also sought to examine the potential metabolic benefits of fermented GB (FGB) with $L$. plantarum CB3 as probiotics and possible distinctions in the degree and/ or mechanism of action compared to GB.

\section{Materials and methods \\ Materials}

Commercial kits for animal tests were purchased from Elabscience (Houston, Texas, USA). Goji berries (Lycium barbarum) were purchased from local markets in Cheongyang Korea. Pectinex Ultra SP-L was obtained from Vision Biochem (Seongnam, Korea). Lactobacillus plantarum CB3, isolated from kimchi in our laboratory, was used as a starter culture for fermentation of goji berries.

\section{Preparation of GB and FGB powder}

For the preparation of GB extract, $10 \mathrm{~g}$ of dried GB was added to $100 \mathrm{~mL}$ of $90{ }^{\circ} \mathrm{C}$ water and extracted for $6 \mathrm{~h}$. Twenty $\mathrm{mL}$ of GB extract was taken and used as a substrate for the production of FGB. Pectinex Ultra SP-L $(0.5 \%, \mathrm{w} / \mathrm{v})$ was added into the GB extract, and the mixture was reacted at $55^{\circ} \mathrm{C}$ for $6 \mathrm{~h}$ with shaking at $160 \mathrm{rpm}$ and sterilized at $100{ }^{\circ} \mathrm{C}$ for $10 \mathrm{~min}$. The sterilized GB suspension was inoculated with $5 \%(\mathrm{v} / \mathrm{v})$ of $L$. plantarum CB3 culture $\left[1 \times 10^{9}\right.$ colony-forming units $(\mathrm{CFU}) / \mathrm{mL}$ ] to give a final cell concentration of about $1 \times 10^{7} \mathrm{CFU} / \mathrm{mL}$, and fermented at $37^{\circ} \mathrm{C}$ for $72 \mathrm{~h}$ with shaking at $160 \mathrm{rpm}$. The GB and FGB suspensions were lyophilized to $88 \%$ solids using an EYELA freeze dryer (FDU-1200, Tokyo Rikakikai Co.) and used for experiments.

\section{Analysis of betaine}

Quantitative analysis of betaine was performed using the method proposed by Lee et al. with some modifications [15]. Betaine from GB and FGB samples (lyophilized to $88 \%$ solids) was detected using a 1260 Infinity system (Agilent Technologies, Santa Clara, CA, USA) attached with an evaporative light scattering detector (ELSD; Agilent Technologies, Santa Clara, CA, USA) and a Discovery C18 column $(4.6 \times 250 \mathrm{~mm}, 5 \mu \mathrm{m}$, SUPELCO, PA, USA). The mobile phase contained water and acetonitrile and was used in isocratic elution as at a 95:5 ratio (v/v \%), respectively. The flow rate of the elution was $0.5 \mathrm{~mL} / \mathrm{min}$ and the evaporator and the nebulizer temperature for the ELSD were 50 and $70{ }^{\circ} \mathrm{C}$, respectively.

\section{Measurement of total phenolics and antioxidant activity}

The total phenolic content of GB and FGB samples (lyophilized to $88 \%$ solids) was determined by modifying the method of previous studies [16]. To determine total phenolic compound and antioxidant activity, lyophilized GB and FGB samples were diluted with $80 \%$ methanol to a concentration of $5 \%$, respectively. For the calibration curve of phenolic compounds, 
gallic acid stock solution $(5 \mathrm{mg} / \mathrm{mL})$ was prepared and diluted with water. Five-hundred microliters of each sample extract was mixed with $300 \mu \mathrm{l}$ of $1 \mathrm{~N}$ Folin-Ciocalteu (Sigma-Aldrich, St. Louis, MO, USA) in a tube. After reacting for $3 \mathrm{~min}$ at room temperature, and $3 \mathrm{~mL}$ of $2 \%$ sodium carbonate was added in the same tube. After reacting at room temperature for $30 \mathrm{~min}$, the absorbance of the mixture was measured at $720 \mathrm{~nm}$ in glass cuvettes using a spectrophotometer (Libra S22, Biochrom Ltd., Cambridge, UK). The same procedure was performed for diluted gallic acid (as a standard). A calibration curve for gallic acid was used to determine the total phenolic contents in the samples, and expressed as gallic acid equivalent (GAE).

The antioxidant activities of GB and FGB samples were determined using diphenylpicrylhydrazyl (DPPH) radical scavenging activity and ferric reducing antioxidant power assay (FRAP). DPPH radical scavenging activity was performed with a $100 \mu \mathrm{M}$ DPPH solution proposed by Liang et al. and Park et al. $[17,18]$. One and half $\mathrm{mL}$ of each methanol extract of GB and FGB samples was dispensed into a tube and added to $1.5 \mathrm{~mL}$ of $100 \mu \mathrm{M}$ DPPH solution. After vortexing and reacting for $30 \mathrm{~min}$ in the dark, absorbance was measured at $517 \mathrm{~nm}$ using a spectrophotometer (Libra S22, biochrom Co.). $80 \%$ of methanol (1.5 mL) and DPPH solution $(1.5 \mathrm{~mL})$ were collected as a control sample. All samples were measured in triplicate. DPPH scavenging activity was calculated using the following equation:

$$
\text { Antioxidant activity }(\%)=1-[(\mathrm{As}-\mathrm{Ab}) / \mathrm{Ac}] \times 100
$$

where antioxidant activity-DPPH radical scavenging activity; $\mathrm{As}, \mathrm{Ab}$ and $\mathrm{Ac}$ represent the absorbance of DPPH with specific samples, blank, and DPPH solutions, respectively.

The FRAP analysis is based on reduction of ferrictripyridyltriazine $\left(\mathrm{Fe}^{3+}{ }_{-} \mathrm{TPTZ}\right)$ complex into ferrous tripyridyltriazine $\left(\mathrm{Fe}^{2+}-\mathrm{TPTZ}\right)$ by interacting with antioxidants in the sample [19]. FRAP assay was performed using the modified Benzie and Strain method [20]. The FRAP reagent was prepared by mixing $300 \mathrm{mM}$ sodium acetate buffer (pH3.6), $10 \mathrm{mM}$ TPTZ solution, and $20 \mathrm{mM} \mathrm{FeCl} 3 \cdot 6 \mathrm{H}_{2} \mathrm{O}$ solution in a ratio of $10: 1: 1(\mathrm{v} / \mathrm{v}) .50 \mu \mathrm{L}$ of each of the methanol extract of GB and FGB was mixed with $1.5 \mathrm{~mL}$ of the FRAP reagent and reacted for $5 \mathrm{~min}$ at room temperature in the dark. Then, the absorbance of the mixtures was measured at $450 \mathrm{~nm}$. The calibration curve was prepared with ferric sulfate and FRAP results for samples are expressed in $\mu \mathrm{g} / \mathrm{mL}$. All samples were measured in triplicate.

\section{Animals and experimental design}

Animals were maintained and handled in accordance with protocols approved by the Institutional Animal Care and Use Committee (Sun Moon University; SM-2020-02-01). Male Sprague-Dawley rats $(\mathrm{n}=8$ / group; 6 weeks old; Samtako Co., Osan, Korea) were individually housed in a controlled environment at $23 \pm 1{ }^{\circ} \mathrm{C}$ at $50 \pm 5 \%$ relative humidity under a $12 \mathrm{~h}$ light/dark cycle. After acclimation for a week on lowfat (LF) diet, animals were split into four weightmatched groups and fed either a low-fat (LF; 10\% kcal as fat), high-fat (HF; $45 \% \mathrm{kcal}$ as fat), or HF diet supplemented with non-fermented (HF/GB) or fermented goji berry at $2 \%(\mathrm{w} / \mathrm{w})$ in diet for 6 weeks (Additional file 1: Table S1). Selection of the $2 \%$ goji berry concentration was based on previously published studies [2123]. Body weight and food intake were measured on a weekly and daily basis, respectively. Food efficiency ratio (FER) was determined as weight gain (g)/energy intake (kcal). After 6 weeks on respective diets, animals were fasted overnight and euthanized by carbon dioxide inhalation. Blood was placed into a sterile Vacutainer plastic tube (BD Vacutainer, Plymouth, UK) and centrifuged at $1000 \times g$ for $10 \mathrm{~min}$ at $4{ }^{\circ} \mathrm{C}$ for serum collection. The liver and visceral fat pads (retroperitoneal and epididymal) were collected and weighed; an adiposity index was determined. Serum and all the tissues were snap-frozen and stored $-80^{\circ} \mathrm{C}$ until analysis.

\section{RNA extraction and quantitative RT-PCR}

Total RNA from liver and epididymal fat tissues was extracted using the RNeasy Mini Kit (Qiagen, Hilden, Germany) per the manufacturer's instructions. cDNAs were synthesized from $2 \mu \mathrm{g}$ of purified RNA samples using TOPscript ${ }^{\mathrm{TM}}$ RT DryMIX (dT18 plus; Enzynomix, Daejeon, Korea) following the manufacturer's protocol. Real-time PCR was performed with the CFX96 Touch $^{\mathrm{TM}}$ Real-Time PCR Detection System (Bio-Rad, Hercules, CA, USA) using ToprealTM qPCR $2 \times$ PreMIX SYBR Green (Enzynomix) for detection. GAPDH was used as a housekeeping gene. Genes of interest were analyzed according to the $2^{-\Delta \Delta C T}$ method [24] and compared with control samples. Primer sequences are provided in Additional file 1: Table S2.

\section{Biochemical analysis}

The levels of serum alkaline phosphatase (ALP) and aspartate aminotransferase (AST) were measured via colorimetric assay kits (ALP: E-BC-K091-S and AST: E-BCK236-M, Elabscience, Houston, TX, USA) according to the manufacturer's guideline. 


\section{Statistical analysis}

Unless stated otherwise (microbiome analysis), statistical analysis was performed by using Prism software (Prism 8.4.3; GraphPad Software, La Jolla, CA, USA). Two-factor repeated-measures analysis of variance (ANOVA) was used to analyze body weight and one-factor ANOVA was performed to analyze the rest of the parameters. Differences between groups were analyzed by using Fisher's least-significant-difference test. Differences were considered significant if $p<0.05$. Data are presented as means \pm standard error of the mean (SEMs).

\section{Results}

\section{Betaine, total polyphenol contents and antioxidant} activities

The concentrations of betaine, total polyphenols and antioxidant activity were measured in GB and FGB samples (lyophilized to $88 \%$ solids) (Table 1). After lyophilization to $88 \%$ solids, the betaine contents of the GB and FGB samples were measured to be $129.29 \mathrm{mg} / \mathrm{mL}$ and $165.51 \mathrm{mg} / \mathrm{mL}$, respectively, indicating that the betaine concentration was increased by about 1.3 -fold by fermentation $(\mathrm{p}<0.05)$.

Total phenolic contents, DPPH radical scavenging ability, and FRAP assay were significantly increased by fermentation $(\mathrm{p}<0.05)$. The total phenolic contents of the GB and FGB were $230.76 \mathrm{mg} / \mathrm{mL}$ and $280.53 \mathrm{mg} / \mathrm{mL}$, respectively, indicating that the total phenolic contents of the GB sample were significantly increased by about 1.2fold that of initial during fermentation. The FRAP assay in the GB and FGB were $273.03 \mu \mathrm{g} / \mathrm{mL}$ and $479.56 \mu \mathrm{g} /$ $\mathrm{mL}$, respectively. This means that the FRAP assay of GB was increased dramatically to about 1.8-fold after fermentation. On the other hand, the DPPH radical scavenging ability of the GB was slightly increased by about $3.6 \%$ after fermentation.

\section{Supplementation of GB and FGB improves hyperphagic phenotypes}

After six weeks on respective diets, all HF fed groups showed significantly (HF, HF/FGB) or partially (HF/GB) higher body weight than the control LF group (LF vs. HF or HF/FGB, $\mathrm{p}<0.05$; LF vs. HF/GB, $\mathrm{p}=0.057$; Fig. 1A).
On the other hand, little difference was observed in cumulative food intake and food efficiency among groups (Fig. 1B, C). Visceral adiposity was evaluated from epididymal and retroperitoneal fat depots (Fig. 1D). HF feeding significantly increased fat mass compared to the LF group (LF vs. HF, p < 0.0001) and little difference was found among HF-fed.

\section{Supplementation of GB and FGB attenuates HF-induced inflammation}

In adipose tissues, both GB and FGB supplementation significantly down-regulated the gene expression of macrophage chemoattactrant protein 1 (MCP-1), as a marker of macrophage infiltration, and pro-inflammatory cytokines, IL- 6 and TNF $\alpha$, compared to the HF group (MCP-1: HF vs. GB or FGB, p < 0.01; IL-1 $\beta$ : HF vs. GB or FGB, $\mathrm{p}<0.05$; TNF $\alpha$ : HF vs. GB or FGB, $\mathrm{p}<0.05$; Fig. 2A-C).

At the systemic level, HF feeding significantly upregulated the gene expression of IL- $1 \beta$ in the liver compared to the LF group, which was suppressed by GB (significantly) and FGB (partially) supplementation (LF vs. HF, $\mathrm{p}<0.01$; HF vs. GB, $\mathrm{p}<0.05$; HF vs. FGB, $\mathrm{p}=0.075$; Fig. 2D). Further, the gene expression of IL-10 as an antiinflammatory cytokine was upregulated by GB and FGB supplementation by $98 \%$ and $97 \%$ compared to the HF group although this did not reach statistical significance (HF vs. GB, $p=0.075$; HF vs. FGB, $p=0.076$; Fig. $2 E$ ).

\section{Supplementation of GB and FGB exerts antioxidant effects}

To investigate the antioxidant properties of GB and FGB supplementation against the HF challenge, the gene expression of antioxidant enzymes was examined. While HF feeding showed little effect on the gene expression of antioxidant enzymes, both GB and FGB supplementation significantly upregulated their gene expression compared to the HF and/or even LF group with more remarkable antioxidant effects by GB supplementation (CAT: LF or HF vs. HF/GB, $\mathrm{p}<0.001$; HF/GB vs. HF/FGB, $\mathrm{p}<0.05$; GPX: LF or HF vs. HF/GB, $\mathrm{p}<0.0001$; HF vs. HF/FGB, $\mathrm{p}<0.01 ; \mathrm{HF} / \mathrm{GB}$ vs. HF/FGB, $\mathrm{p}<0.01 ;$ GSR: LF or HF vs. HF/GB, $\mathrm{p}<0.01$; SOD1: LF or HF vs. HF/GB,

Table 1 Betaine, total phenol contents and antioxidant activities of GB and FGB

\begin{tabular}{lcccc}
\hline Sample & Betaine $(\mathbf{m g} / \mathbf{m L})$ & Total polyphenol $(\mathbf{m g} / \mathbf{m L})$ & DPPH $(\%)$ & FRAP $(\boldsymbol{\mu g} / \mathbf{m L})$ \\
\hline$G B$ & $129.29 \pm 0.49^{\mathrm{b}}$ & $230.76 \pm 0.59^{\mathrm{b}}$ & $64.93 \pm 0.57^{\mathrm{b}}$ & $273.03 \pm 6.40^{\mathrm{b}}$ \\
FGB & $165.51 \pm 1.37^{\mathrm{a}}$ & $280.52 \pm 1.01^{\mathrm{a}}$ & $68.51 \pm 0.68^{\mathrm{a}}$ & $479.56 \pm 6.61^{\mathrm{a}}$
\end{tabular}

Data are expressed as mean \pm SD

Different superscript letters in the same column mean significantly different at $p<0.05$ by Duncan's multiple range test

GB Freeze-dried goji berry sample (88\% solids); FGB Freeze-dried goji berry samples (88\% solids) pretreated with Pectinex Ultra SP-L and fermented with L. plantarum $\mathrm{CB} 3$ 

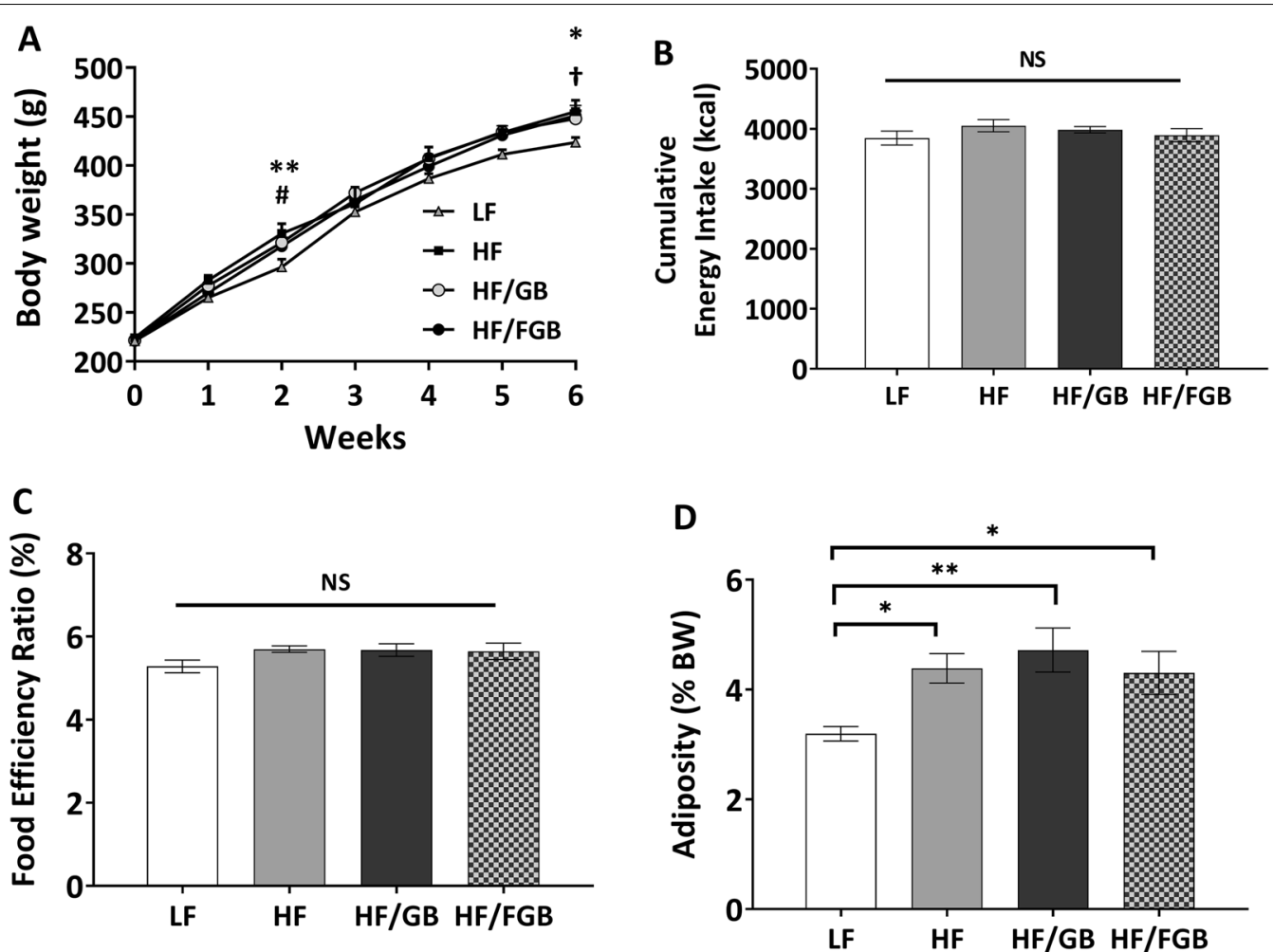

Fig. 1 Supplementation of non-fermented and fermented goji berry has little effects on hyperphagic phenotypes. Body weight (A), cumulative energy intake (B), feed efficiency [weight gain (g)/energy intake (kcal); (C)], and visceral adiposity (D) in rats fed an LF or HF with or without $1 \%$ non-fermented or fermented goji berry supplementation diet for 6 weeks. Values are means \pm SEMs; $n=8 /$ group. For Fig. $1 A$, significant

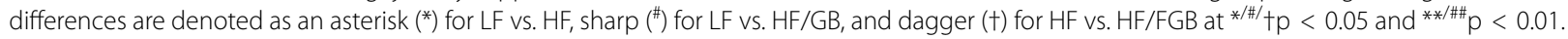
For Fig. 1B-D, significant differences are denoted as an asterisk $\left(^{*}\right)$ at ${ }^{*} \mathrm{p}<0.05$ and ${ }^{* *} \mathrm{p}<0.01$. HF high fat; HF HF without goji berry; HF/FGB HF with fermented goji berry in diet by $2 \%(\mathrm{~W} / \mathrm{W})$; HF/GB HF with non-fermented goji berry in diet by $2 \%(\mathrm{~W} / \mathrm{W})$; LF low fat

$\mathrm{p}<0.0001 ;$ LF vs. HF/FGB, $\mathrm{p}<0.001 ;$ HF vs. HF/FGB, $\mathrm{p}$ $<0.01$; Fig. 3).

\section{Supplementation of GB and FGB improves hepatic functions}

Hepatic functions were examined by the level of ALP and AST in circulation as markers of liver damage (Fig. 4). HF feeding had little effect on the level of serum ALP but the level was significantly decreased by $2 \%$ GB supplementation compared to the HF group (Fig. 4A). The serum ALP level in the HF/GB group was even lower than the LF group by $27 \%$. Similarly, the serum AST level was significantly reduced by both GB and FGB supplementation compared to the HF group (HF vs. GB, p < 0.001; HF vs. FGB, p < 0.05; Fig. 4B).

\section{Supplementation of GB and FGB improves lipid profiles}

In addition to visceral adiposity, lipid profile was further examined in the liver and in circulation (Fig. 5). As a marker of lipogenesis, the gene expression of hepatic peroxisome proliferator-activated receptor gamma
(PPAR $\gamma$ ) was significantly upregulated by HF feeding compared to the LF group, which was normalized to the LF level by both GB and FGB supplementation (LF vs. HF, $\mathrm{p}<0.0001$; HF vs. GB or FGB, $\mathrm{p}<0.0001$; Fig. 5). Similarly, the gene expression of hepatic sterol regulator element-binding protein 1c (SREBP1c), as a marker of adipogenesis, was partially upregulated by HF feeding compared to the LF group and this was significantly suppressed by both GB and FGB supplementation (LF vs. HF, $\mathrm{p}=0.059$; HF vs. GB, $\mathrm{p}<0.01$; HF vs. FGB, $\mathrm{p}<$ 0.001; Fig. 5B). The similar effect of GB and FGB supplementation on lipid profile was also confirmed at the systemic level; the serum TG level was significantly reduced by both GB and RGB supplementation compared to the HF group (HF vs. GB or FGB, p < 0.01; Fig. 5C).

\section{Discussion}

In this present study, we investigated the beneficial effects of GB supplementation on obesity and related metabolic disorders in HF diet-fed rats. We also sought to examine the beneficial effects of fermented GB with L. Plantarum 

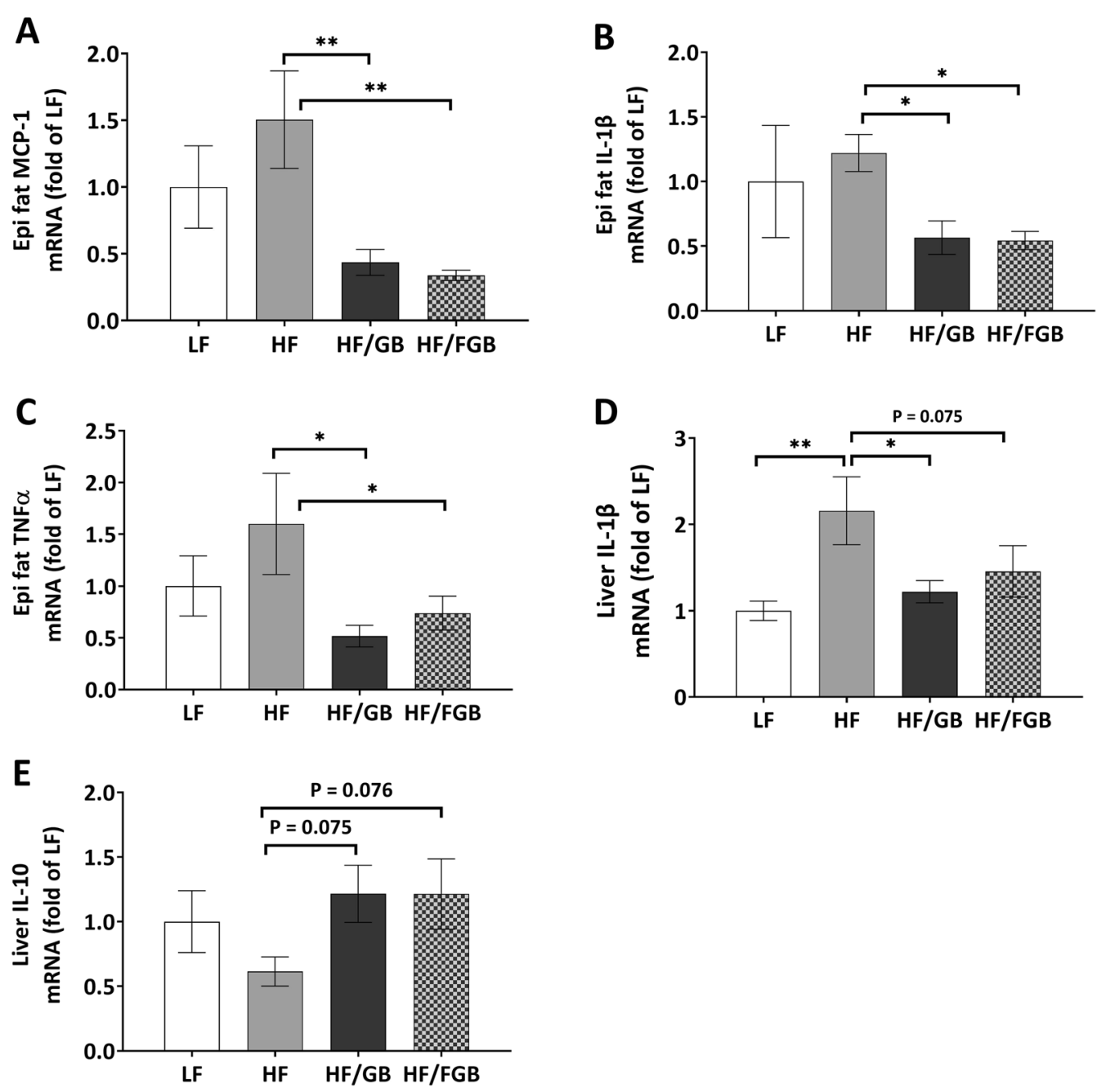

Fig. 2 Supplementation of non-fermented and fermented goji berry attenuates HF-induced inflammation. Gene expression of IL-6 and TNFa in epididymal fat (A-C) and liver (D, E) tissues of rats fed an LF or HF with or without $2 \%$ non-fermented or fermented goji berry supplementation diet for 6 weeks. Values are means \pm SEMs; $n=8$ /group. Significant differences are denoted as an asterisk $\left(^{*}\right)$ at ${ }^{*} p<0.05$ and ${ }^{* *} p<0.01$. HF high fat; HF HF without goji berry; HF/FGB HF with fermented goji berry in diet by $2 \%(\mathrm{w} / \mathrm{w})$; HF/GB HF with non-fermented goji berry in diet by $2 \%$ (w/w); IL interleukin; LF low fat; MCP-1 macrophage chemoattractant protein 1; TNFa tumor necrosis factor alpha

CB3, elucidating the possible metabolic benefits resulting from the fermentation process. Our hypothesis was GB and FGB supplementation as a part of HF diet would improve the HF-induced disturbances in lipid, inflammatory, and oxidative profiles along with hepatic functions, possibly preventing the development of obesity and related metabolic abnormalities, and that these effects would be enhanced by fermentation.

As major bioactive components of GB, concentrations of betaine and total polyphenolic and their corresponding antioxidant activities were measured in GB and FGB samples. Betaine has similar functions to choline, folic acid, vitamin $B_{12}$, and the amino acid methionine known as SAMe (S-adenosylmethionine). In the human body, it functions as a methyl donor and has the effects of improving liver function, cell replication, detoxification, and kidney protection [25, 26].

In this study, we observed that betaine concentration of goji berry was significantly increased by enzyme (Pectinex Ultra SP-L) treatment and L. plantarum CB3 fermentation, confirming the previous finding that the betaine concentration increases after fermenting goji berries with various lactic acid bacteria [27]. Total phenolic contents, DPPH radical scavenging ability, and FRAP assay were significantly increased after fermentation with L. plantarum CB3 inoculation, which is corresponds to the finding of the previous studies [28]. This seems to be because the enzyme and carboxylic acid produced by $L$. 

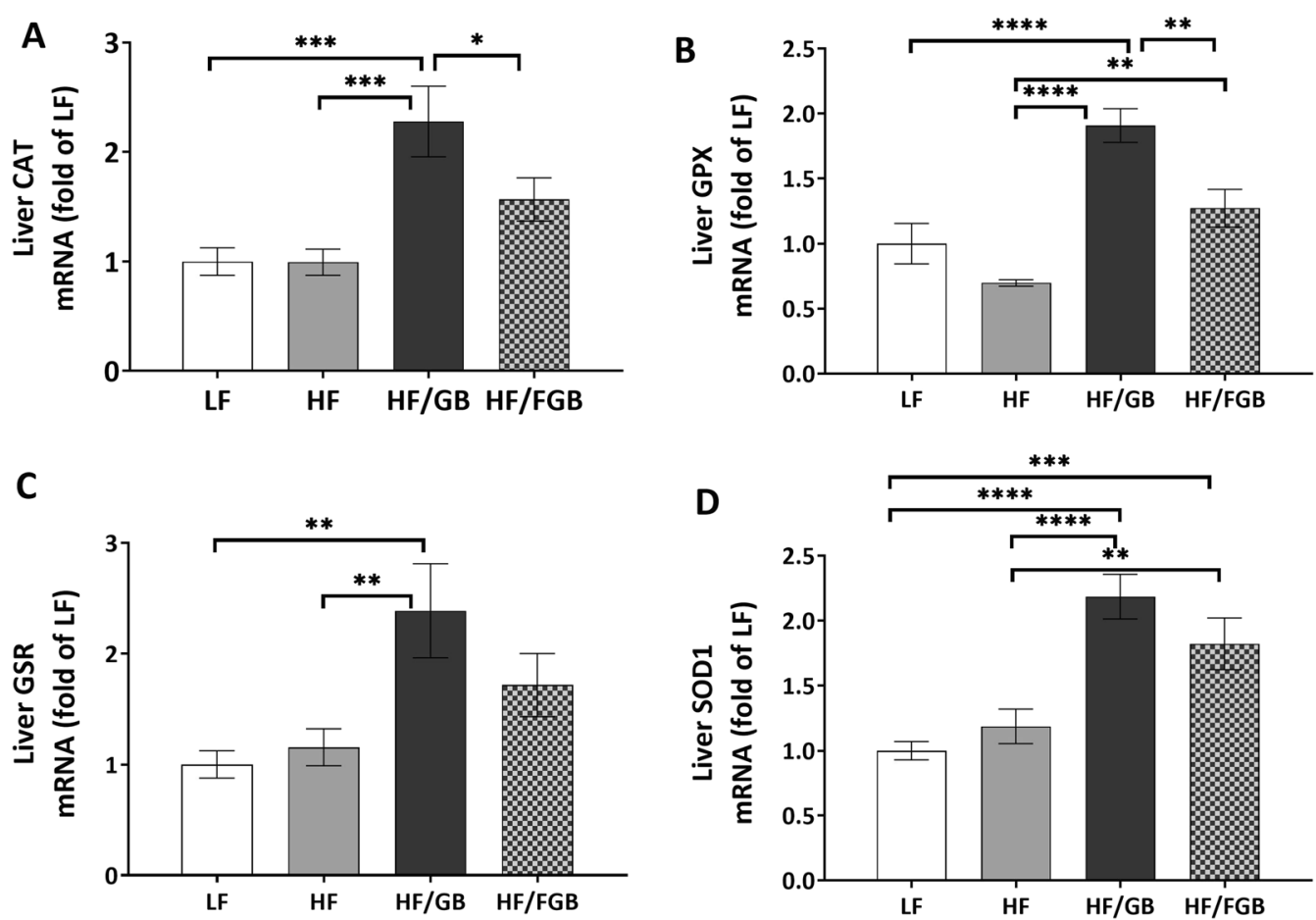

Fig. 3 Supplementation of non-fermented and fermented goji berry exerts antioxidant effects. Gene expression of antioxidant enzymes, CAT (A), GPX (B), GSR (C), and SOD1 (D), in the liver of rats fed an LF or HF with or without 2\% non-fermented or fermented goji berry supplementation diet for 6 weeks. Values are means \pm SEMs; $n=8$ /group. Significant differences are denoted as an asterisk $\left(^{*}\right)$ at ${ }^{*} p<0.05,{ }^{* *} p<0.01,{ }^{* * *} p<0.001$, and ${ }^{* * *} \mathrm{p}<0.0001$. CAT catalase; GPX glutathione peroxidases; GSR glutathione reductase; HF high fat; HF HF without goji berry; HF/FGB HF with fermented goji berry in diet by $2 \%(\mathrm{w} / \mathrm{W})$; HF/GB HF with non-fermented goji berry in diet by $2 \%(\mathrm{w} / \mathrm{w})$; LF low fat; SOD1 superoxide dismutase 1
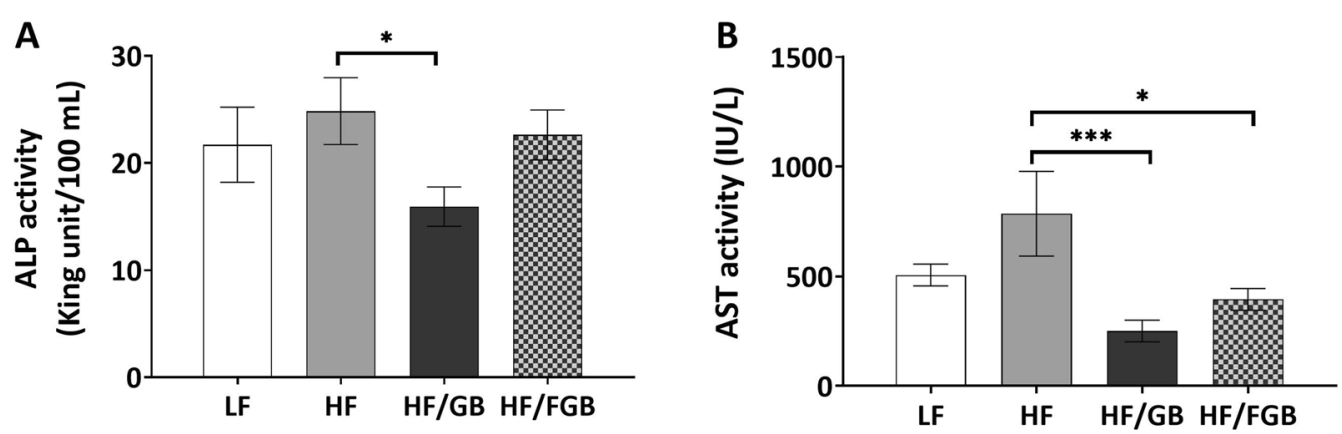

Fig. 4 Supplementation of non-fermented and fermented goji berry protects hepatic functions; levels of serum ALP (A) and AST (B) in rats fed an LF or HF with or without $2 \%$ non-fermented or fermented goji berry supplementation diet for 6 weeks. Values are means \pm SEMs; $n=8 /$ group. Significant differences are denoted as an asterisk $\left(^{*}\right)$ at ${ }^{*} p<0.05$ and ${ }^{* *} p<0.001$. HF high fat; HF HF without goji berry; HF/FGB HF with fermented goji berry in diet by $2 \%(\mathrm{~W} / \mathrm{W})$; HF/GB HF with non-fermented goji berry in diet by $2 \%(\mathrm{w} / \mathrm{W})$. ALP alkaline phosphatase; AST aspartate transaminase; HF/GB HF with non-fermented goji berry in diet by $2 \%(w / w)$; LF low fat

plantarum destroy the cellular structure of GB and cause the release of phenolic substances in the fermentation process [29].

In vivo, $G B$ and $F G B$ supplementation suppressed the gene expression of inflammation indices at the local (MCP-1, IL-1 $\beta$, TNF $\alpha$ in the adipose tissues) and systemic (IL-1 $\beta$ in the liver) levels. In addition, GB and FGB supplementation upregulated the gene expression of antioxidant enzymes, catalase (CAT), glutathione peroxidases (GPX), glutathione reductase (GSR), superoxide dismutase (SOD1) compared to the HF and/or even LF group with more remarkable antioxidant effects by GB 

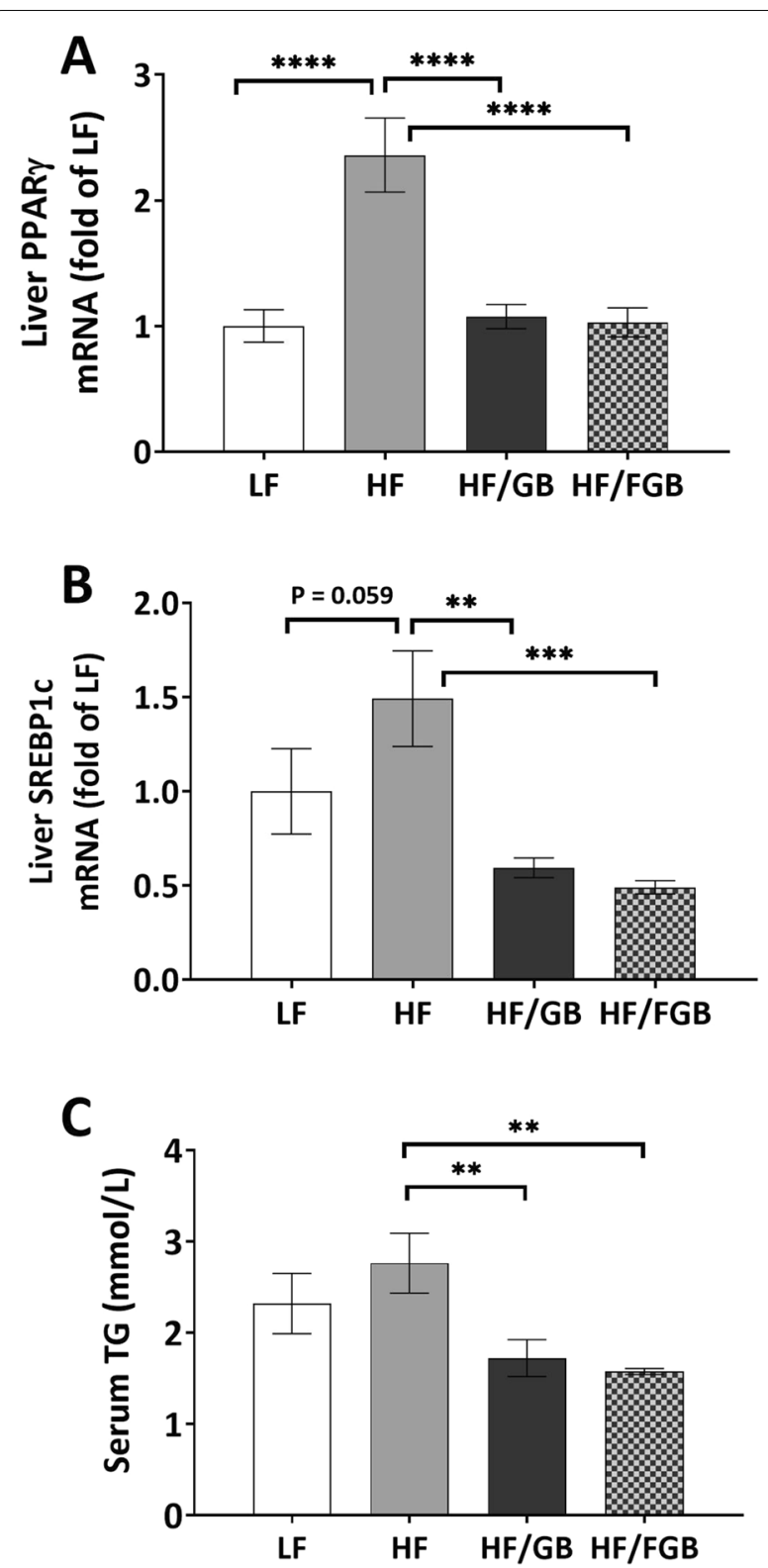

Fig. 5 Supplementation of non-fermented and fermented goji berry improves lipid profiles. Gene expression of PPARY (A) and SREBP1C (B) and serum TG level (C) in rats fed an LF or HF with or without $2 \%$ non-fermented or fermented goji berry supplementation diet for 6 weeks. Values are means \pm SEMs; $n=8$ /group. Significant differences are denoted as an asterisk $\left(^{*}\right)$ at ${ }^{*} p<0.05,{ }^{* *} p<0.01$, ${ }^{* * *} \mathrm{p}<0.001$, and ${ }^{* * *} \mathrm{p}<0.0001$. HF high fat; HF HF without goji berry; HF/FGB HF with fermented goji berry in diet by $1 \%(\mathrm{w} / \mathrm{W})$; $H F / G B H F$ with non-fermented goji berry in diet by $2 \%(\mathrm{w} / \mathrm{w})$; LF low fat; PPARY peroxisome proliferator-activated receptor gamma; SREBP1C sterol regulator element-binding protein $1 c ; T G$ triglyceride

supplementation. Also, GB and FGB supplementation protected from HF-induced damages of the liver and dyslipidemia. Taken together, these data show that both
GB and FGB supplementation improved HF-induced metabolic disturbances and these improvements were associated with their anti-inflammatory and anti-oxidant properties.

It is widely demonstrated that consumption of a HF diet can lead to increases in body weight, energy intake, and visceral fat mass [30]. In this present study, HF feeding for six weeks significantly increased the body weight. While little difference was observed in overall energy intake and feed efficiency between LF and HF fed groups, an increase in adiposity was still observed in HF-fed rats compared to the LF group, confirming the previous finding that total and visceral body fat weights increases proportionally to the level of fat in the diet even when fed isocalorically [31]. It was unexpected that GB and FGB supplementation did not prevent HF-diet induced increases in body weight gain and adiposity. Thus, to better understand the limited effects of supplementation on these obesity phenotypes, we further investigated the related parameters at the molecular level.

The pathogenesis of obesity involves infiltration of macrophages into expanding adipose tissue and the activated macrophages can contribute to local (adipose tissue) and systemic (liver) inflammation, which is primarily promoted by the release of pro-inflammatory cytokines [2]. In particular, studies have demonstrated that HF dietinduced obese animals developed chronic inflammation in adipose and hepatic tissues [32-34]. In this present study, the HF group showed the highest expression of MCP-1 gene, which was remarkably down-regulated by GB and FGB supplementation. Correspondingly, GB and FGB supplementation also exerted anti-inflammatory effects against other pro-inflammatory cytokines, IL-1 $\beta$ and TNF $\alpha$ and in favor of IL-10 as an anti-inflammatory cytokine at the local and systemic levels, which corresponds to the finding of the previous study [35].

The inflammatory cascade facilitates the formation of toxic reactive oxygen species and the subsequent production of oxidative stress, which inhibits the expression of antioxidant enzymes and consequently impairs the antioxidant defense system [3]. Here, we observed that GB and/or FGB supplementation remarkably upregulated the gene expression of antioxidant enzymes in the liver including CAT, GPX, GSR, and SOD1 compared both the LF and HF groups, which is consistent to the previous findings of antioxidant effects of GB and FGB in vitro and in vivo $[10,36,37]$

Excessive oxidative stress also can cause tissue damage to the liver [4]. It is known that ALP and AST enzymes are reliable markers of hepatic function and increased levels of circulating ALP and AST indicate hepatic damages [38, 39]. Here, the highest levels of serum ALP and AST were observed in the HF group and their activities 
were significantly inhibited by GB and/or FGB supplementation. It is important to note that the elevation in these hepatic enzymes have been associated with abnormal hepatic lipid metabolism [5]. Further, studies have previously shown that HF feeding caused ectopic fat deposition, which was closely associated with the upregulated expression of hepatic PPAR $\gamma$ as a transcription factor for adipogenesis [32]. Considered as the master regulator of adipogenesis [40], its overexpression has been known to be sufficient for inducement of hepatic fat deposition [41] in concert with SREBP1c as a transcription factor of lipogenesis and also a mediator of PPAR $\gamma$ expression [42]. In this study, both GB and FGB significantly suppressed HF-induced upregulation of PPAR $\gamma$ and SREBP1c gene expression in the liver. This was also confirmed at the systemic level. As a well-known indicator of obesity, dyslipidemia is primarily characterized by the elevation of triglycerides in circulation [6]. In this present study, the serum TG level was increased in the HF group by $19 \%$ compared to the LF group and this elevation was significantly suppressed by both $G B$ and FGB supplementation. The beneficial effects of GB supplementation also has been reported in previous studies via inhibiting the expression of hepatic lipogenesis factors and/or decreasing the level of triglyceride in circulation, which confirms the findings of this study $[43,44]$.

Taken together, it can be confirmed that GB and FGB supplementation improved HF-induced metabolic dysregulation in adipose and liver tissues at the molecular level primarily by enhancing anti-inflammatory and antioxidant responses.

There are some considerations to this study that deserve more attention. First, we employed a HF regime for six weeks to induce diet-induced obesity and related metabolic disturbances. This protocol was sufficient to induce obese phenotypes (increases in body weight and visceral fat mass), which was further supported by HFinduced dysregulation of hepatic metabolism (IL-1 $\beta$, PPAR $\gamma$, and SREBP1c). However, we failed to find any remarkable differences between the LF and HF groups for the other metabolic parameters at the molecular level including those for inflammation, antioxidant actions, and hepatic functions. Thus, a study with longer duration than six weeks would better enable the establishment of other measures for the development of HF-induced obesity. In the meanwhile, despite little difference the LF and HF groups in those metabolic parameters, both GB and FGB supplementation demonstrated metabolic benefits compared to the HF group, even compared to the LF group for the regulation of the antioxidant defense system. Notably, these beneficial effects were more outstanding in the HF/GB group. Previous studies have reported different degree and/or mechanism for metabolic effects between non-fermented and fermented prebiotics resulting from fermentation with probiotics [45-47]. In these studies, metabolic benefits were more enhanced or different mechanism of action was resulted by fermentation compared to the original prebiotics. In this regard, it is possible that different pathways might have been involved in metabolic benefits observed in the HF/FGB group. Thus, further investigation into multiple possible metabolic pathways would identify the potential mechanism of action by FGB for its metabolic benefits. Further, since the gut microbiota has been identified the primary target by prebiotics and probiotics for their metabolic benefits, metagenomic and metabolomic analyses would provide a better understanding of distinct mechanism of action on metabolic profiles between GB and FGB.

In conclusion, we demonstrated that GB and FGB supplementation protected from HF-induced metabolic complications primarily by improving hepatic function and corresponding lipid metabolism via their anti-inflammatory and antioxidant properties. To our knowledge, this is the first in vivo study confirming metabolic benefits of GB in a fermented form. Thus, these findings support the potential application of both GB and FGB to ameliorate obesity-associated metabolic abnormalities.

\section{Abbreviations \\ ALP: Alkaline phosphatase; AST: Aspartate transaminase; ANOVA: Analysis of variance; CAT: Catalase; GPX: Glutathione peroxidases; GSR: Glutathione reduc- tase; HF: High fat; HF/FGB: HF with fermented goji berry in diet by $2 \%(\mathrm{w} / \mathrm{w})$; HF/GB: HF with non-fermented goji berry in diet by $2 \%(\mathrm{w} / \mathrm{W})$; IL: Interleukin; LF: Low fat; PPARY: Peroxisome proliferator-activated receptor gamma; ROS: Reactive oxygen species; SOD1: Superoxide dismutase; SREBP1c: Sterol regula- tor element-binding protein 1c; TG: Triglyceride; TNFa: Tumor necrosis factor alpha; MCP-1: Macrophage chemoattractant protein.}

\section{Supplementary Information}

The online version contains supplementary material available at https://doi. org/10.1186/s13765-021-00642-1.

Additional file 1: Table S1. Composition of experimental diets. Table S2. Primer sequences used for RT-PCR.

\section{Acknowledgements \\ This research was supported by the Ministry of Trade, Industry and Energy in 2019 [Grant Number P0010311]. \\ Authors' contributions \\ Conceptualization: HS (Hee-jae Suh), HP, SL. Animal experiment: SL, SJ, YP, CY, UH. Sample preparation: SJ, YP, HS (Hyunji Seo), CY, UH. Sample analysis: SL, SJ. Data analysis: SL. Writing, review, and editing: HS (Hee-jae Suh), HP, SL. Funding acquisition: HS (Hee-jae Suh). All authors have read and approved the final manuscript.}

\section{Declarations}

Competing interests

The authors declare that there is no competing interests. 
Received: 27 May 2021 Accepted: 9 September 2021

Published online: 21 September 2021

\section{References}

1. Pedersen SD (2013) Metabolic complications of obesity. Best Pract Res Clin Endocrinol Metab 27:179-193

2. Longo M, Zatterale F, Naderi J, Parrillo L, Formisano P, Raciti GA, Beguinot F, Miele C (2019) Adipose tissue dysfunction as determinant of obesityassociated metabolic complications. Int J Mol Sci. https://doi.org/10. 3390/ijms20092358

3. Fernández-Sánchez A, Madrigal-Santillán E, Bautista M, Esquivel-Soto J, Morales-González Á, Esquivel-Chirino C, Durante-Montiel I, SánchezRivera G, Valadez-Vega C, Morales-González JA (2011) Inflammation, oxidative stress, and obesity. Int J Mol Sci 12:3117-3132

4. Cichoż-Lach H, Michalak A (2014) Oxidative stress as a crucial factor in liver diseases. World J Gastroenterol WJG 20:8082

5. Chen SC-C, Tsai SP, Jhao J-Y, Jiang W-K, Tsao CK, Chang L-Y (2017) Liver fat, hepatic enzymes, alkaline phosphatase and the risk of incident type 2 diabetes: a prospective study of 1,32,377 adults. Sci Rep 7:1-9

6. Klop B, Elte JWF, Cabezas MC (2013) Dyslipidemia in obesity: mechanisms and potential targets. Nutrients 5:1218-1240

7. Kulczyński B, Gramza-Michałowska A (2016) Goji berry (Lycium barbarum): composition and health effects-a review. Polish J Food Nutr Sci 66:67-76

8. Ming M, Guanhua L, Zhanhai Y, Guang C, Xuan Z (2009) Effect of the Lycium barbarum polysaccharides administration on blood lipid metabolism and oxidative stress of mice fed high-fat diet in vivo. Food Chem 113:872-877

9. Yang F-L, Wei Y-X, Liao B-Y, Wei G-J, Qin H-M, Pang X-X, Wang J-L (2020) Effects of Lycium barbarum polysaccharide on endoplasmic reticulum stress and oxidative stress in obese mice. Front Pharmacol 11:742

10. Amagase $\mathrm{H}$, Sun B, Borek C (2009) Lycium barbarum (goji) juice improves in vivo antioxidant biomarkers in serum of healthy adults. Nutr Res 29:19-25

11. Yang Y, Li W, Li Y, Wang Q, Gao L, Zhao J (2014) Dietary Lycium barbarum polysaccharide induces Nrf2/ARE pathway and ameliorates insulin resistance induced by high-fat via activation of PI3K/AKT signaling. Oxid Med Cell Longev. https://doi.org/10.1155/2014/145641

12. Bianchi F, Larsen $N$, de Mello TT, Adorno MAT, Kot W, Saad SMI, Jespersen $L$, Sivieri K (2018) Modulation of gut microbiota from obese individuals by in vitro fermentation of citrus pectin in combination with Bifidobacterium longum BB-46. Appl Microbiol Biotechnol 102:8827-8840

13. Kim B-G, Choi S-Y, Kim M-R, Suh HJ, Park HJ (2010) Changes of ginsenosides in Korean red ginseng (Panax ginseng) fermented by Lactobacillus plantarum M1. Process Biochem 45:1319-1324

14. Jung J, Jang HJ, Eom SJ, Choi NS, Lee N-K, Paik H-D (2019) Fermentation of red ginseng extract by the probiotic Lactobacillus plantarum KCCM 11613P: ginsenoside conversion and antioxidant effects. J Ginseng Res 43:20-26

15. Lee S-M, Park C-K, Cho B-G, Cho K-S, Min B-S, Bae K-H (2011) A convenient HPLC/ELSD method for the quantitative analysis of betaine in Lycium chinense Nat Prod Sci 17:104-107

16. Negi PS, Jayaprakasha GK, Jena BS (2003) Antioxidant and antimutagenic activities of pomegranate peel extracts. Food Chem 80:393-397

17. Park Y-J, Hwang U, Park S, Sim S, Jeong S, Park M, Kang M, Lee Y, Song Y, Park H (2021) Optimal bioconversion for compound K production from red ginseng root (CA Mayer) by sequential enzymatic hydrolysis and its characteristics. Appl Biol Chem 64:1-11

18. Liang X-L, Wang X-L, Li Z, Hao Q-H, Wang S-Y (2010) Improved in vitro assays of superoxide anion and 1, 1-diphenyl-2-picrylhydrazyl (DPPH) radical-scavenging activity of isoflavones and isoflavone metabolites. J Agric Food Chem 58:11548-11552

19. Spiegel M, Kapusta K, Kołodziejczyk W, Saloni J, Żbikowska B, Hill GA, Sroka Z (2020) Antioxidant activity of selected phenolic acids-ferric reducing antioxidant power assay and QSAR analysis of the structural features. Molecules 25:3088

20. Benzie IFF, Strain JJ (1996) The ferric reducing ability of plasma (FRAP) as a measure of "antioxidant power": the FRAP assay. Anal Biochem 239:70-76
21. Tian B, Zhang Z, Zhao J, Ma Q, Liu H, Nie C, Ma Z, An W, Li J (2021) Dietary whole goji berry (Lycium barbarum) intake improves colonic barrier function by altering gut microbiota composition in mice. Int J Food Sci Technol 56:103-114

22. Crepaldi LD, Mariano IR, Trondoli AJPC, Moreno FN, Piovan S, Formigoni M, Salgueiro-Pagadigorria CL, de Godoi VAF (2018) Goji berry (Lycium barbarum) extract improves biometric, plasmatic and hepatic parameters of rats fed a high-carbohydrate diet. J Pharm Pharmacol 6:877-889

23. Kang Y, Xue Y, Du M, Zhu M-J (2017) Preventive effects of goji berry on dextran-sulfate-sodium-induced colitis in mice. J Nutr Biochem 40:70-76

24. Livak KJ, Schmittgen TD (2001) Analysis of relative gene expression data using real-time quantitative PCR and the $2^{-\triangle \Delta C T}$ method. Methods 25:402-408

25. Barak AJ, Tuma DJ (1983) Betaine, metabolic by-product or vital methylating agent? Life Sci 32:771-774

26. Chambers ST (1995) Betaines: their significance for bacteria and the renal tract. Clin Sci 88:25-27

27. Lee H-C, Lee B-C, Kim S-D, Lee K-S, Paik S-W, Lee S-S, Kim S-M (2008) Changes in composition of Gugija (Lycii fructus) species according to harvest time. Korean J Med Crop Sci 16:306-312

28. Wang M, Ouyang X, Liu Y, Liu Y, Cheng L, Wang C, Zhu B, Zhang B (2021) Comparison of nutrients and microbial density in goji berry juice during lactic acid fermentation using four lactic acid bacteria strains. J Food Process Preserv 45:e15059

29. Feng L, Tang N, Liu R, Nie R, Guo Y, Liu R, Chang M (2021) Effects of different processing methods on bioactive substances and antioxidation properties of Lycium barbarum (goji berry) from China. Food Biosci. https://doi org/10.1016/j.fbio.2021.101048

30. Buettner R, Schölmerich J, Bollheimer LC (2007) High-fat diets: modeling the metabolic disorders of human obesity in rodents. Obesity 15:798-808

31. Boozer CN, Schoenbach G, Atkinson RL (1995) Dietary fat and adiposity: a dose-response relationship in adult male rats fed isocalorically. Am J Physiol 268:E546-E550

32. Gao M, Ma Y, Liu D (2015) High-fat diet-induced adiposity, adipose inflammation, hepatic steatosis and hyperinsulinemia in outbred CD-1 mice. PLOS ONE 10:e0119784. https://doi.org/10.1371/journal.pone.0119784

33. Jeong S, Park M, Kang M, Park Y-J, Lee M, You C, Hwang U, Song Y, Park $H$, Lee $S$ (2021) Supplementation of non-fermented and fermented red ginseng improves obese phenotypes, lipid and inflammatory profiles, and antioxidant defense system in high fat-fed rats. J Food Nutr Res 9:154-162

34. Lee S, Goodson M, Vang W, Kalanetra K, Barile D, Raybould H (2020) 2'-fucosyllactose supplementation improves gut-brain signaling and diet-induced obese phenotype and changes the gut microbiota in high fat-fed mice. Nutrients 12:1003

35. Ávila CN, Trindade FMR, Penteado JO, Janke F, Schneider JP, Uecker JN, Rincón JAA, de Barros CC, Andreazza R, Pieniz S (2020) Anti-inflammatory effect of a goji berry extract (Lycium barbarum) in rats subjected to inflammation by lipopolysaccharides (LPS). Braz Arch Biol Technol 63:31

36. Liu Y, Cheng H, Liu H, Ma R, Ma J, Fang H (2019) Fermentation by multiple bacterial strains improves the production of bioactive compounds and antioxidant activity of goji juice. Molecules 24:3519

37. Nardi GM, Januario AGF, Freire CG, Megiolaro F, Schneider K, Perazzoli MRA, Do Nascimento SR, Gon AC, Mariano LNB, Wagner G (2016) Antiinflammatory activity of berry fruits in mice model of inflammation is based on oxidative stress modulation. Pharmacognosy Res 8:S42

38. Cui Y, Yang X, Lu X, Chen J, Zhao Y (2014) Protective effects of polyphenols-enriched extract from Huangshan Maofeng green tea against CCl4induced liver injury in mice. Chem Biol Interact 220:75-83

39. Ren $D, H u Y$, Luo Y, Yang $X$ (2015) Selenium-containing polysaccharides from Ziyang green tea ameliorate high-fructose diet induced insulin resistance and hepatic oxidative stress in mice. Food Funct 6:3342-3350

40. Nerurkar PV, Lee Y-K, Nerurkar VR (2010) Momordica charantia (bitter melon) inhibits primary human adipocyte differentiation by modulating adipogenic genes. BMC Complement Altern Med 10:1-10

41. Yu S, Matsusue K, Kashireddy P, Cao WQ, Yeldandi V, Yeldandi AV, Rao MS, Gonzalez FJ, Reddy JK (2003) Adipocyte-specific gene expression and adipogenic steatosis in the mouse liver due to peroxisome 
proliferator-activated receptor gamma1 (PPARgamma1) overexpression. J Biol Chem 278:498-505. https://doi.org/10.1074/jbc.M210062200

42. Kim JB, Spiegelman BM (1996) ADD1/SREBP1 promotes adipocyte differentiation and gene expression linked to fatty acid metabolism. Genes Dev 10:1096-1107

43. Peng Y, Ma C, Li Y, Leung KS-Y, Jiang Z-H, Zhao Z (2005) Quantification of zeaxanthin dipalmitate and total carotenoids in Lycium fruits (Fructus lycii). Plant Foods Hum Nutr 60:161-164

44. de Souza Zanchet MZ, Nardi GM, de Oliveira SBL, Filippin-Monteiro FB, Locatelli C (2017) Lycium barbarum reduces abdominal fat and improves lipid profile and antioxidant status in patients with metabolic syndrome. Oxid Med Cell Longev. https://doi.org/10.1155/2017/9763210

45. Morales P, Barros L, Dias MI, Santos-Buelga C, Ferreira ICFR, Asquieri ER, Berrios JDJ (2016) Non-fermented and fermented jabuticaba (Myrciaria cauliflora Mart.) pomaces as valuable sources of functional ingredients. Food Chem 208:220-227
46. Yeap SK, Mohd Ali N, Mohd Yusof H, Alitheen NB, Beh BK, Ho WY, Koh SP, Long K (2012) Antihyperglycemic effects of fermented and nonfermented mung bean extracts on alloxan-induced-diabetic mice. J Biomed Biotechnol. https://doi.org/10.1155/2012/285430

47. Park CH, Kim M, Woo M, Lee KH, An BK, Yokozawa T, Song YO (2018) Comparison of the effects of nonfermented and fermented Panax ginseng root against hypertriglycemia in high-fat diet-fed mice. J Med Food 21:317-321

\section{Publisher's Note}

Springer Nature remains neutral with regard to jurisdictional claims in published maps and institutional affiliations.

\section{Submit your manuscript to a SpringerOpen ${ }^{\circ}$ journal and benefit from:}

- Convenient online submission

- Rigorous peer review

- Open access: articles freely available online

- High visibility within the field

- Retaining the copyright to your article

Submit your next manuscript at $\boldsymbol{\nabla}$ springeropen.com 